\title{
Protective effect and mechanism of glutaredoxin 1 on coronary arteries endothelial cells damage induced by high glucose
}

\author{
Shuyan $\mathrm{Li}^{\mathrm{a}}$, Yan Sun ${ }^{\mathrm{b}}$, Xiaodan $\mathrm{Qi}^{\mathrm{c}}$, Yan $\mathrm{Shi}^{\mathrm{a}}$, Han $\mathrm{Gao}^{\mathrm{a}}$, Qi Wu ${ }^{\mathrm{c}}$, Xiucai Liu ${ }^{\mathrm{a}}$, Haitao Yu ${ }^{\mathrm{d}, *}$ \\ and Chunjing Zhang, \\ ${ }^{a}$ Department of Biochemistry, Qiqihar Medical University, Qiqihar 161006, China \\ ${ }^{b}$ Department of Clinical Pathogenic Microorganism, Qiqihar Medical University, Qiqihar 161006, \\ China \\ ${ }^{c}$ Department of Clinical Biochemistry, Qiqihar Medical University, Qiqihar 161006, China \\ ${ }^{d}$ Department of Biology Genetics, Qiqihar Medical University, Qiqihar 161006, China
}

\begin{abstract}
In recent years, diabetes and its associated complications have become a major public health concern. The cardiovascular risk increases significantly in diabetes patients. It is a complex disease characterized by multiple metabolic derangements and is known to impair cardiac function by disrupting the balance between pro-oxidants and antioxidants at the cellular level. The subsequent generation of reactive oxygen species (ROS) and accompanying oxidative stress are hallmarks of the molecular mechanisms responsible for cardiovascular disease. Protein thiols act as redox-sensitive switches and are believed to be a key element in maintaining the cellular redox balance. The redox state of protein thiols is regulated by oxidative stress and redox signaling and is important to cellular functions. The potential of the thiol-disulfide oxidoreductase enzymes (thioredoxin and glutaredoxin systems) in defense against oxidative stress has been noted previously. Increasing evidence demonstrates that glutaredoxin 1 (Grxl), a cytosolic enzyme responsible for the catalysis of protein deglutathionylation, plays distinct roles in inflammation and apoptosis by inducing changes in the cellular redox system. This study investigates whether and how Grx1 protects coronary artery vascular endothelial cells against high glucose (HG) induced damage. Results indicate that the activation of eNOS/NO system is regulated by Grx 1 and coupled with inhibition of JNK and NF- $\mathrm{kB}$ signaling pathway which could alleviate the oxidative stress and apoptosis damage in coronary arteries endothelial cells induced by HG.
\end{abstract}

Keywords: Grx 1, diabetes cardiovascular complications, redox homeostasis, eNOS/NO system, redox signaling pathway

\footnotetext{
*Corresponding authors: Haitao Yu, Department of Biology Genetics, Qiqihar Medical University, Qiqihar 161006, China. Tel.: +86 452 2663711; Fax: +86 452 2663711; E-mail: yht422@126.com.

Chunjing Zhang, Department of Biochemistry, Qiqihar Medical University, Qiqihar 161006, China. Tel.: +86 452 2663711; Fax:+86 452 2663711; E-mail: cjzhang2005@163.com.
}

0959-2989/14/\$27.50 @ 2014 - IOS Press and the authors. 


\section{Introduction}

Vascular complication in diabetes is closely related to the damage of vascular endothelial cell form and function in the hyperglycemic state. Proliferation, differentiation and apoptosis of cardiomyocyte and vascular endothelial cells are regulated by many factors inside and outside of the cells, and change of redox homeostasis is especially important for cell survival and death.

Glutaredoxin 1 (Grx 1) is the primary enzyme responsible for catalysis of the thiol-disulfide oxidoreduction reactions, especially the regulation of glutathionylation, S-nitrosylation and decarbonyl ation of proteins [1-8]. The effect of Grx1 is essential for developing novel therapeutic approaches for treating diseases involving dysregulated apoptosis, such as cancer, heart disease, diabetes, and neurodegenerative diseases, where alterations in redox homeostasis are hallmarks for pathogenesis.

According to our previous study, after providing exogenous Grx 1, the oxidation protein and apoptosis could be antagonized in the human umbilical vein endothelial cells and $\mathrm{H} 9 \mathrm{c} 2$ cardiomyocyte induced by high glucose (HG). The mRNA expression of Grx 1 was downregulated under HG in primary cultured porcine coronary arteries endothelial cells. However, protective effect and mechanism of exogenous Grx 1 on vascular endothelial cells damage induced by HG have not yet elaborated. This study discovered that Grx 1 can alleviate the oxidative stress and apoptosis damage of vascular endothelial cells via regulation of the activation of eNOS/NO system and coupling with the inhibition of JNK/NF- $\mathrm{KB}$ signaling pathway activation.

\section{Materials and methods}

\subsection{Materials}

Grx 1 (ProSpec, Israel), OxyBlot ${ }^{\mathrm{TM}}$ protein oxidation detection kit (Millipore, S7150 USA \& Canada), NO assay kit (Nanjing Jiancheng, China), Annexin V and PI fluorescein staining kit (Bender MedSystems, Austria), anti-p-eNOS, p-JNK and p-NF- $\mathrm{BB}($ Cell Signaling Technology and Santa Cruz Biotechnology), RIPA Lysis Buffer System (Santa Cruz Biotechnology), Anti-active Caspase 3 and Anti-GAPDH antibody (Abcam, Britain). Pierce ECL Western Bloting Substrate (Thermo, USA).

\subsection{Culture of coronary artery endothelial cells}

Fresh porcine coronary arteries were obtained, after collagenase digestion under aseptic conditions, primary porcine coronary arteries endothelial cells were obtained and incubated in the Dulbecco's Modified Eagle Medium (DMEM) medium supplemented with $10 \%$ fetal bovine serum in a humidified atmosphere with $5 \% \mathrm{CO}_{2}$ at $37^{\circ} \mathrm{C}$.

\subsection{Oxidative modification of proteins analysis}

Oxidative modified proteins were measured using the OxyBlot protein oxidation detection kit. Follow the instruction of Kit: briefly, prepare protein solution, derivatize the carbonyl groups, separate the protein sample by SDS-PAGE, transfer to Polyvinylidene Fluoride (PVDF) membrane (Bio-Rad, USA), block non-specific sites by using 1\% Bull Serum Albumin (BSA), incubate in primary and HRP-conjugated secondary antibody, add Chemiluminescent reagent and then expose to film. 


\subsection{Annexin V and PI binding assay}

Annexin V and PI fluorescein staining kit (Bender MedSystems, Austria) were utilized to measure cell apoptosis by following the manufacturer's instruction. Cells were washed and suspended in $200 \mu \mathrm{l}$ $1 \times$ binding buffer $\left(10 \mathrm{mmol} / \mathrm{L}\right.$ HEPES pH $\left.7.4,140 \mathrm{mmol} / \mathrm{L} \mathrm{NaCl}, 2.5 \mathrm{mmol} / \mathrm{L} \mathrm{CaCl}{ }_{2}\right) / 1 \times 10^{6} / \mathrm{L}$ cells. Cells were then incubated with Annexin V (1:20) for 3 min followed by incubation with propidium iodide (PI, $1 \mathrm{mg} / \mathrm{ml}$ ) for $15 \mathrm{~min}$. Apoptosis rate was evaluated by flow cytometry.

\subsection{Western blot analysis}

Cultured cells were harvested and lysed with RIPA lysis buffer. Protein samples were electrophoresed on SDS-polyacrylamide gels. The proteins in the gel were transferred onto PVDF membrane. The membranes were blocked in $5 \%(\mathrm{w} / \mathrm{v})$ nonfat dry milk and then reacted with primary antibodies of anti-active Caspase 3, p-eNOS and p-JNK, p-NF- $\mathrm{kB}$, and HRP-labeled antibody. Proteins in the membrane were then visualized using the enhanced chemiluminescence detection kit. Meanwhile, with the reduced glyceraldehyde-phosphate dehydrogenase (GAPDH) as the loading control, western bolt results were photographed and analyzed by using ChemiDoc MP System (ChemiDocTMXRS, American Bio-rad Company).

\subsection{Statistical analysis}

Data was presented as means $\pm \mathrm{S}$. Comparisons between two groups were analyzed by a two-tailed Student $t$ test, A value of $p<0.05$ was considered to be significant.

\section{Results}

\subsection{Grx 1 protects cells against $H G$ induced oxidative modification of proteins}

OxyBlot assay was performed to detect the carbonyl groups introduced into proteins and regulation

(A)

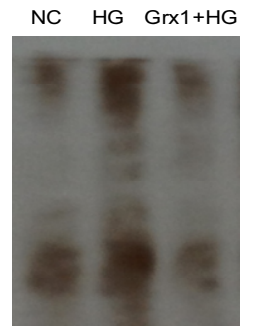

(B)

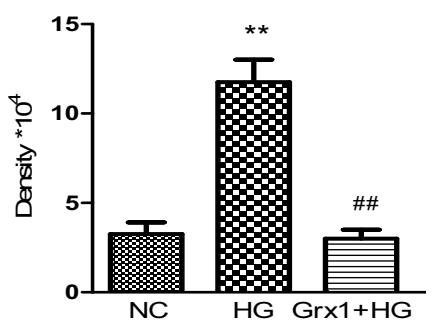

Fig. 1. Oxidative modification of proteins detection. The Oxyblot protein oxidation detection kit was used to analyze the oxidation protein expression level. The reaction procedures were conducted according to the manufacturer's instructions. PCAECs were treated with/without $25 \mathrm{mmol} / \mathrm{L}$ D-glucose for $24 \mathrm{~h}$. (A) Detection of oxidatively modified proteins (protein carbonyls) (B) Histogram analysis of the Density.

Note: ${ }^{*} \mathrm{P}<0.01$ compared with $\mathrm{NC} ;{ }^{\#} \mathrm{P}<0.01$, compared with $\mathrm{HG}$. NC: normal glucose $5.5 \mathrm{mmol} / \mathrm{L}$, HG: high glucose $25 \mathrm{mmol} / \mathrm{L}$, Grx 1: Glutaredoxin $5 \mu \mathrm{g} / \mathrm{mL}$. 
effect of Grx 1 in the porcine coronary arteries endothelial cells (PCAECs) induced by HG. The results (see Figure 1) showed that oxidative modification of protein was increased by treatment with HG in cells, which could be inhibited by pre-treatment with Grx 1. The data indicate that Grx 1 system is involved in the redox regulation of certain enzyme activities, and this system protects cells from HG induced damage by regulating the redox state.

\subsection{Grx 1 suppresses $H G$ induced cells apoptosis}

To investigate the role of Grx 1 in porcine coronary arteries endothelial cell apoptosis induced by HG, cell apoptotic ratio was assayed with flow cytometry and cleaved Caspase 3 express level was detected by using western blot. The results (see Figure 2 ) showed that early apoptosis rate increased extremely, $17 \mathrm{kDa}$ subunit of active/cleaved Caspase 3 level was obviously activated in HG group, however, which could be significantly reduced after providing Grx 1 .

\subsection{Grx 1 reverses $H G$ decreased $p$-eNOS and NO level}

Activation of eNOS/NO system was reported to be involved in diabetic vascular damage. In the study, the effect of Grx 1 on NO level was observed and p-eNOS expression was detected by western blot in porcine coronary arteries endothelial cells induced by HG. The results (see Figure 3) showed that secretion of NO was transient, which increased at $12 \mathrm{~h}$, and then decreased at 24 and $48 \mathrm{~h}$ compared with the control group. The expression of p-eNOS in HG $(25-50 \mathrm{mmoL} / \mathrm{L})$ group was significantly down-regulated during 12-24 h. After Grx 1 treatment, NO level was restored; p-eNOS

(A)
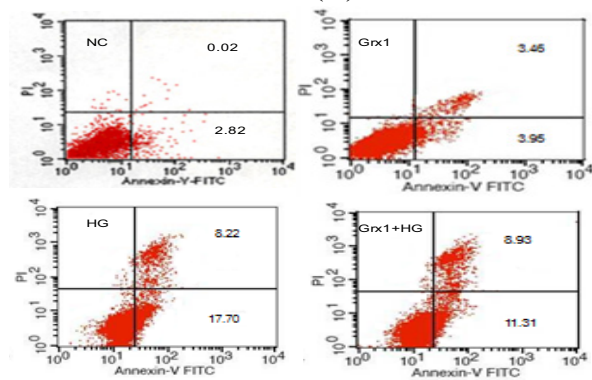

(C) NC $\quad H G \quad$ Grx1 Grx1+HG
(B)

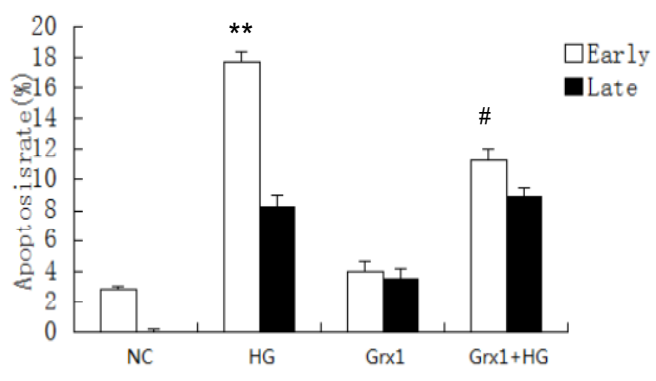

(D)
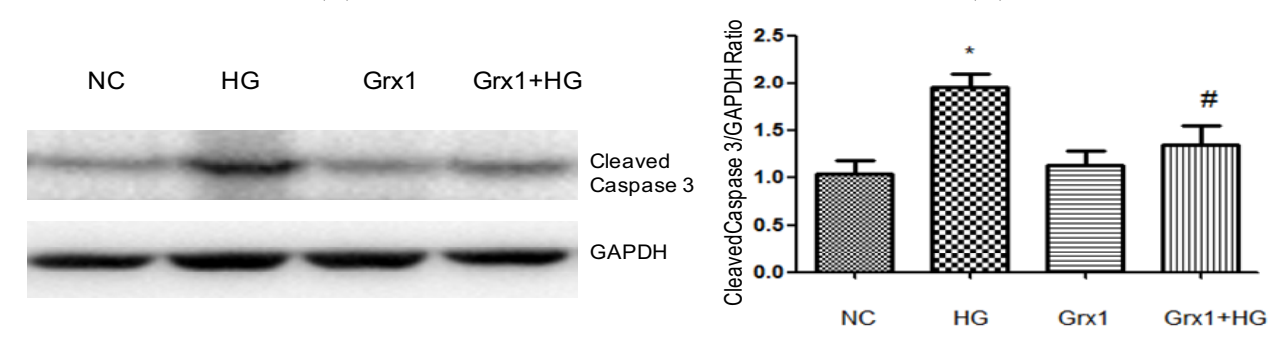

Fig. 2. Apoptosis rates and cleaved Caspase 3 express level analysis. (A, B) Apoptosis rates were analyzed by flow cytometry; (C,D) Western blot and histogram analysis of Caspase 3 proteins expression level after pre-treatment with Grx 1 in PCAECs induced by $\mathrm{HG}$ for $24 \mathrm{~h}$.

Note: ${ }^{* *} \mathrm{P}<0.01$ compared with $\mathrm{NG} ;{ }^{*} \mathrm{P}<0.05$, compared with $\mathrm{HG}$. NC: normal glucose $5.5 \mathrm{mmol} / \mathrm{L}, \mathrm{HG}$ : high glucose 25 $\mathrm{mmol} / \mathrm{L}$, Grx 1: Glutaredoxin $5 \mu \mathrm{g} / \mathrm{mL}$. 
(A)

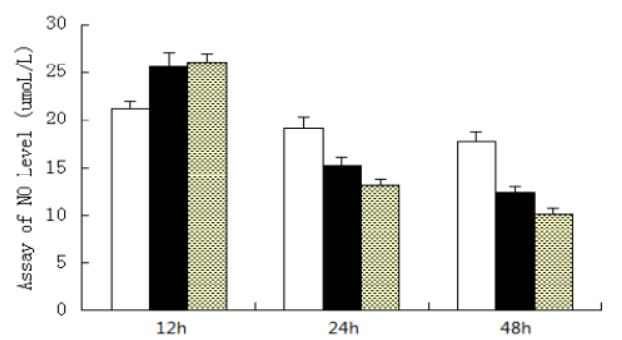

(C)

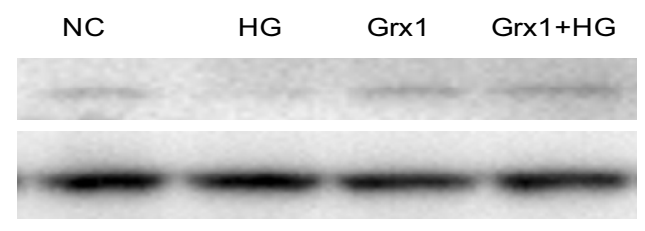

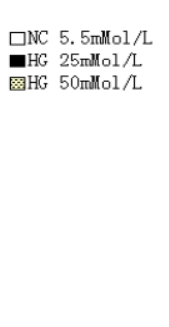

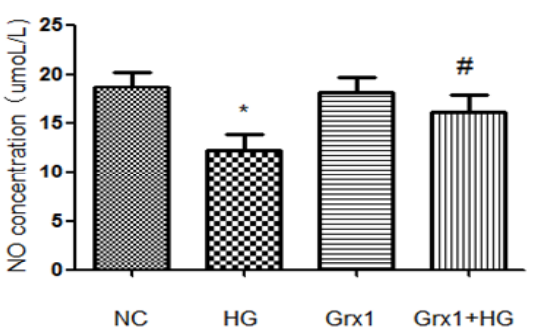

(D)

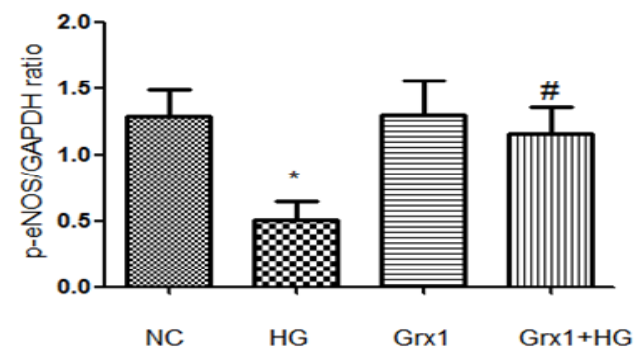

Fig. 3. Activation of eNOS/NO system analysis. (A, B) Assay of NO level by using NO assay kit in PCAECs induced by HG for $48 \mathrm{~h}$; (C,D) Western blot and Histogram analysis of p-eNOS proteins expression level after pre-treatment with Grx 1 in PCAECs induced by HG for $24 \mathrm{~h}$.

Note: $* \mathrm{P}<0.05$ compared with $\mathrm{NC}$; ${ }^{\#} \mathrm{P}<0.05$, compared with HG. NC: normal glucose $5.5 \mathrm{mmol} / \mathrm{L}$, HG: high glucose 25 $\mathrm{mmol} / \mathrm{L}$, Grx 1: Glutaredoxin $5 \mu \mathrm{g} / \mathrm{mL}$.

level was turned over compared with HG group. The data indicate that Grx 1 can reverse NO generation and eNOS phosphorylation level, which are inhibited by $25 \mathrm{mmol} / \mathrm{L}$ HG for 24-48 hours in porcine coronary arteries endothelial cells.

\subsection{Grx 1 antagonist $H G$ induced activation of $J N K / N F-\kappa B$ signaling pathway}

To further prove that JNK and NF- $\mathrm{KB}$ apoptosis signaling pathway can be regulated by Grx 1 in vascular endothelial cell induced by HG, western bolt analysis was used to detect phosphorylation of apoptosis signaling protein $\mathrm{JNK}$ and $\mathrm{NF}-\mathrm{kB}$ in this study. The results (see Figure 4) showed that PCAECs were exposed to HG for $1-2 \mathrm{~h}$, the phosphorylation level of NF- $\mathrm{BB}$ and JNK increased significantly, but it decreased significantly in Grx 1 pre-protection group.

\section{Discussion}

The damage caused by diabetes is mainly due to its complications, and the development mechanism of chronic complications in diabetes is very complicated and unclear.

It has been reported that a decrease in NO resulting from decreased eNOS, may partially contribute to the predisposition of the female aorta to injury early in diabetes [9]. Our present data suggest that the reduction of $\mathrm{NO}$ and p-eNOS, the increase of oxidative modification of protein, early apoptosis rate and cleaved Caspase 3 expression could be detected in primary cultured porcine coronary arteries endothelial cells induced by HG. The above results are consistent with the reported results that HG 
(A)

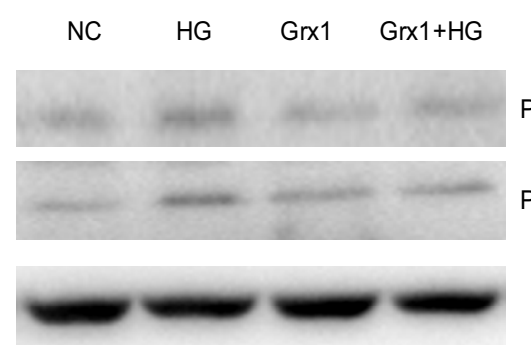

(B)

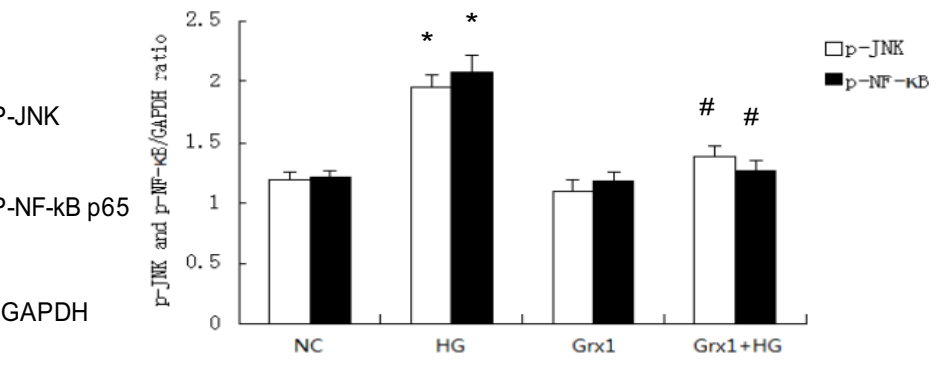

Fig. 4. Analysis of $p-\mathrm{JNK}$ and $p-\mathrm{NF}-\kappa \mathrm{B}$ p65 expression level .(A) Western blot analysis of proteins expression level after pre-treatment with Grx 1 in PCAECs induced by HG for 1h; (B) Histogram analysis of the density.

Note: ${ }^{*} P<0.05$ compared with NG; ${ }^{\sharp} P<0.05$, compared with HG. NC: normal glucose $5.5 \mathrm{mmol} / \mathrm{L}$, HG: high glucose 25 $\mathrm{mmol} / \mathrm{L}$, Grx 1: Glutaredoxin $5 \mu \mathrm{g} / \mathrm{mL}$.

could induce reactive oxygen species generation and reduce levels of NO and p-eNOS, which leads to diabetic mice endothelium functional imbalance [10,11]. By contrast, after providing Grx 1 preprotection, NO generation and p-eNOS level increase in a concentration dependent manner, and oxidative modification of protein, early apoptosis rate and cleaved Caspase 3 expression level decrease significantly. The results are in agreement with recent reports that Grx 1/GSH could maintain the activity of eNOS to promote the release of $\mathrm{NO}$ and restore vascular function via de-glutathionylation and de-nitration $[1,12]$.

The activation of JNK signaling pathway is the major mechanism of cardiomyocyte damage induced by HG $[13,14]$. In our previous study, the mechanism of antioxidant carnosine antagonizing myocardial apoptosis induced by HG was found to be correlated with p-JNK / p-c-Jun expression [15]. Recent study suggested that Grx 1-mediated IKK $\beta$ Cys-179 de-glutathionylation could play an important role in diabetic complications via regulating the autocrine and paracrine of proinflammatory cytokine [16]. However, the precise mechanisms of redox regulation in the Grx 1-mediated signal pathways have not been clarified in vascular endothelial cell induced by HG. In the present study, we are interested in the possible involvement of JNK and NF- $\mathrm{KB}$ apoptosis signaling pathway in the Grx 1-mediated anti-apoptotic effect against oxidative stress in vascular endothelial cell. The study found that Grx 1 could inhibit vascular endothelial cell apoptosis via modulation of the JNK and NF-kappaB signaling cascade in primary cultured porcine coronary arteries endothelial cells exposed to HG. These are consistent with the results that Grxl gene therapy could prevent the activation of death signaling pathway JNK and p38, and activate survival signaling pathway Akt-FoxO signal network to restore the myocardial function of diabetes ischemia reperfusion rats, and is also consistent with the result that Grx 1-mediated glutathionylation has protective effect on anti-inflammation mechanism [17-19].

In conclusion, our data support that the protective role of Grx 1 in regulating the progression of diabetic vascular diseases in porcine coronary arteries endothelial cells induced by HG and the effect is mediated through regulation of oxidative stress, enhancing the activity of eNOS and promoting the release of $\mathrm{NO}$, accompanying the suppression of $\mathrm{JNK}$ and $\mathrm{NF}-\mathrm{kB}$ signal pathway. Based on these findings, vascular endothelial damage induced by $\mathrm{HG}$ could be effectively decreased to protect vascular function in diabetic via improving the antioxidant system function of Grx 1/GSH and regulating the redox homeostasis. 


\section{Acknowledgement}

This work was funded by grants from Committee of Education Science Foundation of Heilongjiang Province (No. 12541902 and No. 12541905), the Foundation of Qiqihar Science and Technology Bureau and National Natural Science Foundation of China (No. 31240006).

\section{References}

[1] C. Inadomi, H. Murata and Y. Ihara, Overexpression of glutaredoxin protects cardio myocytes against nitric oxideinduced apoptosis with suppressing the S-nitrosylation of proteins and nuclear translocation of GAPDH, Biochemical and Biophysical Research Communications 425 (2012), 656-661.

[2] J. Lu and A. Holmgren, The thioredoxin antioxidant system, Free Radic. Biol. Med. 66 (2014), 75-87.

[3] E.M. Allen and J.J. Mieyal, Protein-thiol oxidation and cell death: Regulatory role of glutaredoxins, Antioxidant \& Redox Signal 17 (2012), 1748-1763.

[4] P. Ghezzi, Protein glutathionylation in health and disease, Biochimica et Biophysica Acta 1830 (2013), 3165-3172.

[5] M.L. De Benedetto, C.R. Capo and A. Ferri, Glutaredoxin 1 is a major player in copper metabolism in neuroblastoma cells, Biochim. Biophys. Acta. 1840 (2014), 255-261.

[6] Y. Du, H. Zhang and S. Montano, Plasma glutaredoxin activity in healthy subjects and patients with abnormal glucose levels or overt type 2 diabetes, Acta Diabetol. 51 (2014), 225-232.

[7] C.M. Wong, L. Marcocci, D. Das, X.H. Wang, H.B. Luo, M. Zungu-Edmondsona and Y.J. Suzuki, Mechanism of protein decarbonylation, Free Radical Biology and Medicine 65 (2013), 1126-1133.

[8] M.M. Gallogly, D.W. Starke and J.J. Mieyal, Mechanistic and kinetic details of catalysis of thiol-disulfide exchange by glutaredoxins and potential mechanisms of regulation, Antioxid. and Redox Signal 11 (2009), 1059-1081.

[9] X.Y. Han, R. Zhang, L. Anderson and R. Rahimian, Sexual dimorphism in rat aortic endothelial function of streptozotocin-induced diabetes: Possible involvement of superoxide and nitric oxide production, Eur. J. Pharmacol. 723 (2014), 442-450.

[10] J. Sun, Y. Pu and P. Wang, TRPV1-mediated UCP2 upregulation ameliorates hyperglycemia-induced endothelial dysfunction, Cardiovasc. Diabetol. 12 (2013), 69.

[11] Y.X. Kang, M.H. Hu and Y.H. Zhu, Antioxidative effect of the herbal remedy and its active component tetramethylpyrazine on high glucose-treated endothelial cells, Life Sciences 84 (2009), 428-436.

[12] C.A. Chen, F.D. Pascali, A. Basye, C. Hemann and J. Zweier, Redox modulation of endothelial nitric oxide synthase by Glutaredoxin-1 through reversible oxidative post-translational modification, Biochemistry 52 (2013), 6712-6723.

[13] C.J. Li, L. Lv and H. Li, Cardiac fibrosis and dysfunction in experimental diabetic cardiomyopathy are ameliorated by alpha-lipoic acid, Cardiovasc. Diabetol. 11 (2012), 73.

[14] W. Yu, J.L. Wu and F. Cai, Curcumin alleviates diabetic cardiomyopathy in experimental diabetic rats, PLoS One 7 (2012), e52013.

[15] X.D. Qi, C.J. Zhang and Y. Shi, Carnosine inhibits cardiomyocyte apoptosis via modulation of the JNK and NF-kappaB signaling cascade in H9c2 cells exposed to high glucose, Chin. J. Biochem. Mol. Biol. 30 (2014), 58-64.

[16] M.D. Shelton, Glutaredoxin regulates autocrine and paracrine proinflammatory responses in retinal glial (müller) cells, J. Biol. Chem. 284 (2009), 4760-4766.

[17] I. Lekli, S. Mukherjee and D. Ray, Functional recovery of diabetic mouse hearts by Glutaredoxin-1 gene therapy: Role of Akt-FoxO signaling network, Gene. Ther. 17 (2010), 478-485.

[18] P.S. Tsou, V. Addanki and J.A. Haas, Role of glutaredoxin-mediated protein S-glutathionylation in cellular nitroglycerin tolerance, J. Pharmacol. Exp. Ther. 329 (2009), 649-656.

[19] B.C. Liao, C.W. Hsieh and Y.C. Lin, The glutaredoxin/glutathione system modulates NF-kappaB activity by glutathionylation of p65 in cinnamaldehyde-treated endothelial cells, Toxicol. Sci. 116 (2010), 151-163. 